\title{
Serum Elafin in acne patients with and without post acne scar
}

A.M.El-Refaei ${ }^{1}$, S.H.Ahmed ${ }^{1}$, N.A.Abd Elhafiez ${ }^{2}$ and M.A.Elgndy ${ }^{1}$

${ }^{1}$ Dermatology, Andrology and Venerology Dept., Faculty of Medicine, Benha Univ., Benha, Egypt

${ }^{2}$ Clinical pathology Dept., Faculty of Medicine, Benha Univ., Benha, Egypt

E-Mail:mohamed@gmail.com

\section{Abstract}

Acne vulgaris (AV) is an inflammatory skin disease of the pilosebaceous unit. There are numerous factors in the etiopathogenesis of AV. The main factors are inflammation, abnormal keratinization, microbial flora changes, and increased sebum production. The sequence in which these events occur is still not certain, but recently, inflammation has been suggested as the initial factor. The aim was to estimate elafin levels in the serum of moderate and severe acne cases versus controls and to investigate if elafin and can be used as markers for diseases severity. Methods: 80 patients were included in this study, Patients under study were subjected to the following: Full history taking, General examination, Dermatological clinical examination, and Laboratory investigations: Elafin using enzyme-linked immunosorbent assay (ELISA) technique. Results: Patients showed significantly higher Elafin levels when compared to control group $(\mathrm{p}<0.001)$. In addition, paients with severe acne showed significantly higher Elafin levels when compared to moderate subgroups ( $\mathrm{p}=0.005,<0.001$ respectively), Conclusion: Using marker levels as elafin in determining acne severity will help in overcoming the limitations and disadvantages of CRP performance. Elafin acts as an alarm anti protease; the measurement of its levels in serum of patients with acne vulgaris provides a tool for monitoring disease activity and could be used as an indicator of the disease severity and inflammatory state.

Keywords: Elafin, Acne, Post acne scar.

\section{Introduction}

Acne vulgaris (AV) is an inflammatory skin disease of the pilosebaceous unit. There are numerous factors in the etiopathogenesis of AV. The main factors are inflammation, abnormal keratinization, microbial flora changes, and increased sebum production. The sequence in which these events occur is still not certain, but recently, inflammation has been suggested as the initial factor. The mechanisms behind the beginning and maintenance of the inflammatory response are not fully known, but Propionibacterium acnes (P.C) plays an important role in these mechanisms [1].

Propionibacterium acnes triggers inflammation both directly and indirectly; it increases proinflammatory cytokine release and antimicrobial peptide expression. Propionibacterium acnes also stimulates the innate immune response by activating Toll-like receptor (TLR)-2. Sebocytes are the major cells within sebaceous glands that constitute the pilosebaceous unit along with hair, hair follicles, and the arrector pili muscles. Along with the keratinocytes, sebocytes act as immune cells [2].

Acne vulgaris is one of the most common skin diseases and mainly affects patients between the age of 10 to 20 . It was previously reported that development of acne scars are generally associated with moderate to severe inflammatory acne lesions. These scars can cause not only cosmetic problems but also psychological ones, which may lead to the poor quality of life to the patient [3].

Acne scars are classified into atrophic and hypertrophic types, and most of them are in the form of atrophic lesions. Therefore, many researchers focused on the treatment and analysis of atrophic scars rather than analyzing acne hypertrophic scars [4].

Although there were some studies about hypertrophic scars, the underlying pathogenesis is still unclear and there are only a few studies focusing on acne hypertrophic scars. Until now, it is generally believed that excessive collagen accumulation and prolonged inflammation contribute to the formation of hypertrophic scars [5].

Elafin, is one of natural Antimicrobial peptides (AMPs), and it is a serine protease inhibitor produced by epithelial and immune cells with anti-inflammatory properties [6]. Elafin's functions are not only restricted to its protease inhibitor action but also extend to involve other molecular properties, influencing cellular proliferation and inflammation [7].

The aim of the study was to estimate elafin levels in the serum of moderate and severe acne cases versus controls and to investigate if elafin and C-reactive protein (CRP) can be used as markers for diseases activity and if they can be used to predict post acne scarring or not.

\section{Patients and methods}

This is a case-control study. Which was approved by the Local Ethics Committee of Research involving human subjects in Benha Faculty of Medicine. Informed consents were obtained from all participants before sample collection. The study was done during the period from $1 / 8 / 2019$ to $15 / 12 / 2019$

It was conducted in the Dermatology outpatient clinic, Faculty of Medicine, Benha University Hospital. The study included 80 participants; 40 patients suffering from moderate and severe acne with or with out acne scars, in addition to 40 healthy individuals , age , BMI and sex matched individuals as a control group.

This study included 40 patients with moderate to severe acne. In addition, 40 apparently normal age, sex and BMI matched individuals were included as a 
control subjects' group. Acne patients was further classified into 2 groups:

- Group I: This group included 16 patients with out acne scars.

- Group П: This group included 24 patients with acne scars.

The severity was graded according to the global acne grading system (GAGS) score.

\subsection{Exclusion criteria}

- History of systemic or topical anti-acne therapy previous month before the study.

- Infectious, inflammatory, autoimmune systemic, cutaneous diseases or conditions other than acne such as rheumatic disease, chronic infections, cancer, sleep disorders, diabetes mellitus, polycystic ovary syndrome, metabolic syndrome, hyperlipidemia, and intake of estrogen/progesterone pills, statins, fibric acid derivatives or niacin.

- Patients with acne fulminans and acne conglobata will be excluded from the study.

Patients under study were subjected to the following: Full history taking , General examination, Dermatological clinical examination, and Laboratory investigations: Estimation of serum levels of CReactive protein (CRP) using latex agglutination technique and Elafin using enzyme-linked immunosorbent assay (ELISA) technique.

\subsection{Statistical analysis}

The collected data was revised, coded, tabulated and introduced to a PC using Statistical package for Social Science (IBM Corp. Released 2011. IBM SPSS Statistics for Windows, Version 20.0. Armonk, NY: IBM Corp.). Data were presented and suitable analysis was done according to the type of data obtained for each parameter. Descriptive statistics: Mean, Standard deviation $( \pm$ SD) for numerical data. Frequency and percentage of non-numerical data. Shapiro test was done to test the normality of data distribution. Analytical statistics: Student T Test was used to assess the statistical significance of the difference between two study group means. For the comparison of the three groups' means, one way analysis of variance (ANOVA) was used. Chi-Square test was used to examine the relationship between two qualitative variables. Fisher's exact test: was used to examine the relationship between two qualitative variables when the expected count is less than 5 in more than $20 \%$ of cells. Correlation analysis: To assess the strength of association between two quantitative variables. The correlation coefficient defines the strength and direction of the linear relationship between two variables. The ROC Curve (receiver operating characteristic) provides a useful way to evaluate the sensitivity and specificity for quantitative diagnostic measures that categorize cases into one of two groups. The optimum cut off point was defined as that which maximized the AUC value. The area under the ROC curve (AUC) results were considered excellent for AUC values between 0.9-1, good for AUC values between 0.8-0.9, fair for AUC values between 0.7-0.8, poor for AUC values between 0.6-0.7 and failed for AUC values between 0.5-0.6. Regression analysis: Logistic and ordinal regression analyses were used for prediction of risk factors, using generalized linear models. $p$ is significant if $<0.05$ at confidence interval $95 \%$.

\section{Results}

The present study included 40 patients with acne vulgaris, and 40 healthy control groups. All cases were stratified according to GAGs score into moderate (16), while (24) had severe acne.

The mean age of acne vulgaris patients and control group were $21.2 \pm 3.7$ and $21.7 \pm 4.1$ years respectively. More than half of the acne patient were female(65\%), while (50\%)in the control group. the mean BMI of patient group and control group were $24.7 \pm 3.5$ and $23.2 \pm 1.21$ respectively. Threre was no significant differences between both studied groups regarding age,gendre and $\mathrm{BMI}(\mathrm{p}<0.05)$ Table (1).

Table (1) Comparison of demographic and anthropometric data between all studied groups and subgroups.

\begin{tabular}{|c|c|c|c|c|c|c|c|}
\hline & & \multirow[b]{2}{*}{$\begin{array}{c}\text { Control } \\
\mathrm{N}=40\end{array}$} & \multicolumn{3}{|c|}{ Acne Vulgaris } & \multirow[b]{2}{*}{$\mathbf{P}^{1}$} & \multirow[b]{2}{*}{$\mathbf{P}^{2}$} \\
\hline & & & $\begin{array}{l}\text { Total } \\
\text { N=40 }\end{array}$ & $\begin{array}{c}\text { Moderate } \\
\mathbf{N}=16\end{array}$ & $\begin{array}{c}\text { Severe } \\
\mathrm{N}=24\end{array}$ & & \\
\hline \multirow{2}{*}{ Age (years) } & Mean & 21.7 & 21.2 & 22.0 & 20.6 & \multirow{2}{*}{0.532} & \multirow{2}{*}{0.254} \\
\hline & $\pm \mathrm{SD}$ & \pm 4.1 & \pm 3.7 & \pm 4.9 & \pm 2.5 & & \\
\hline \multirow{2}{*}{ Male } & $\mathrm{N}$ & 20 & 14 & 3 & 11 & \multirow{4}{*}{0.175} & \multirow{4}{*}{0.079} \\
\hline & $\%$ & $50 \%$ & $35 \%$ & $18.8 \%$ & $45.8 \%$ & & \\
\hline \multirow{2}{*}{ Female } & $\mathrm{N}$ & 20 & 26 & 13 & 13 & & \\
\hline & $\%$ & $50 \%$ & $65 \%$ & $81.3 \%$ & $54.2 \%$ & & \\
\hline D & Mean & 23.2 & 24.7 & 25.1 & 24.4 & \multirow{2}{*}{0.211} & \multirow{2}{*}{0.516} \\
\hline BIVII $\left(\mathrm{kg} / \mathrm{m}^{-}\right)$ & $\stackrel{ \pm}{\mathrm{SD}}$ & \pm 1.2 & \pm 3.5 & \pm 4.8 & \pm 2.2 & & \\
\hline
\end{tabular}

SD, standard deviation; P1, comparison of total cases versus control; p2, comparison of moderate and severe acne. 
No significant differences were found between moderate and severe acne groups regarding family history and smoking $(\mathrm{p}<0.05)$ There was a significant difference between patient with moderate and severe acne regarding the risk factors (sun exposure and stress) (P>0.05), Fig (1).

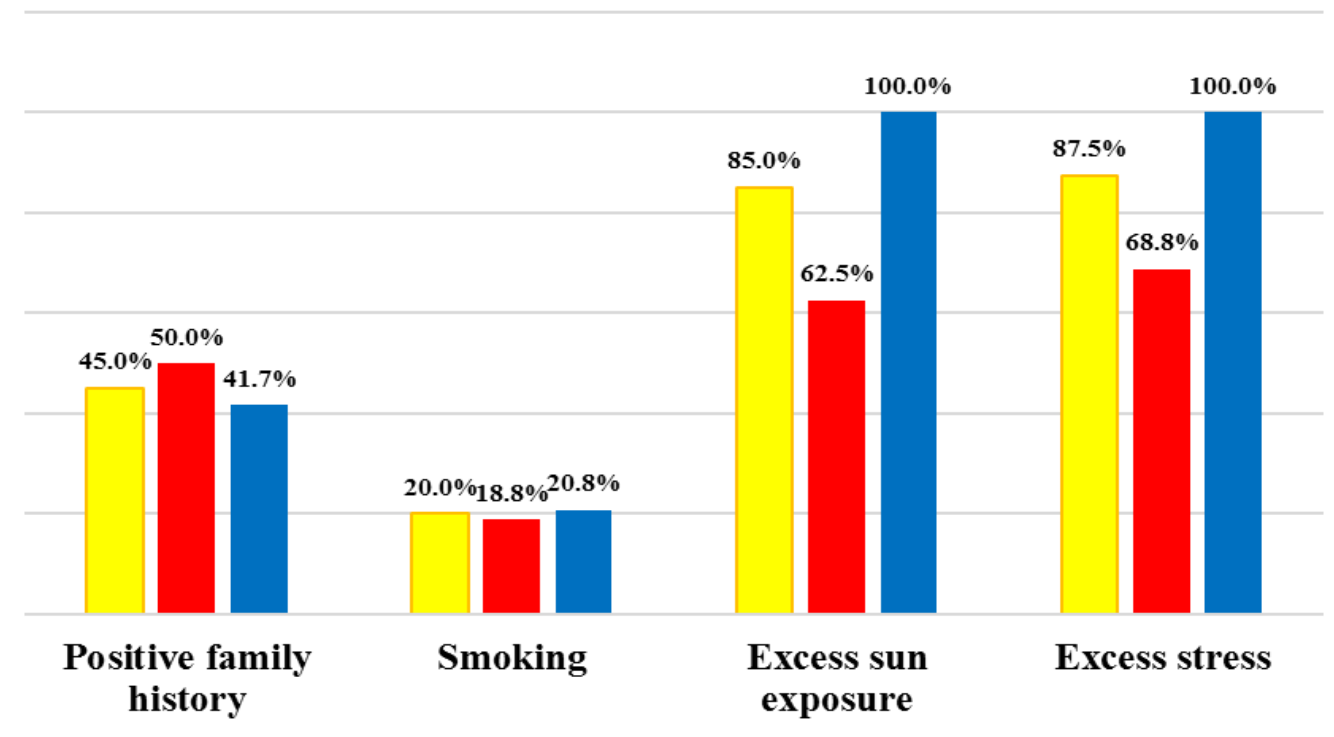

$\square$ Total AV $\square$ Moderate $\square$ Severe

Fig (1) risk factors in all acne patient.

Patients showed significantly higher Elafin levels when compared to control group $(\mathrm{p}<0.001)$. In addition, paients with severe acne showed significantly higher Elafin levels when compared to moderate subgroups $(p=0.005,<0.001$ respectively), Fig (2).

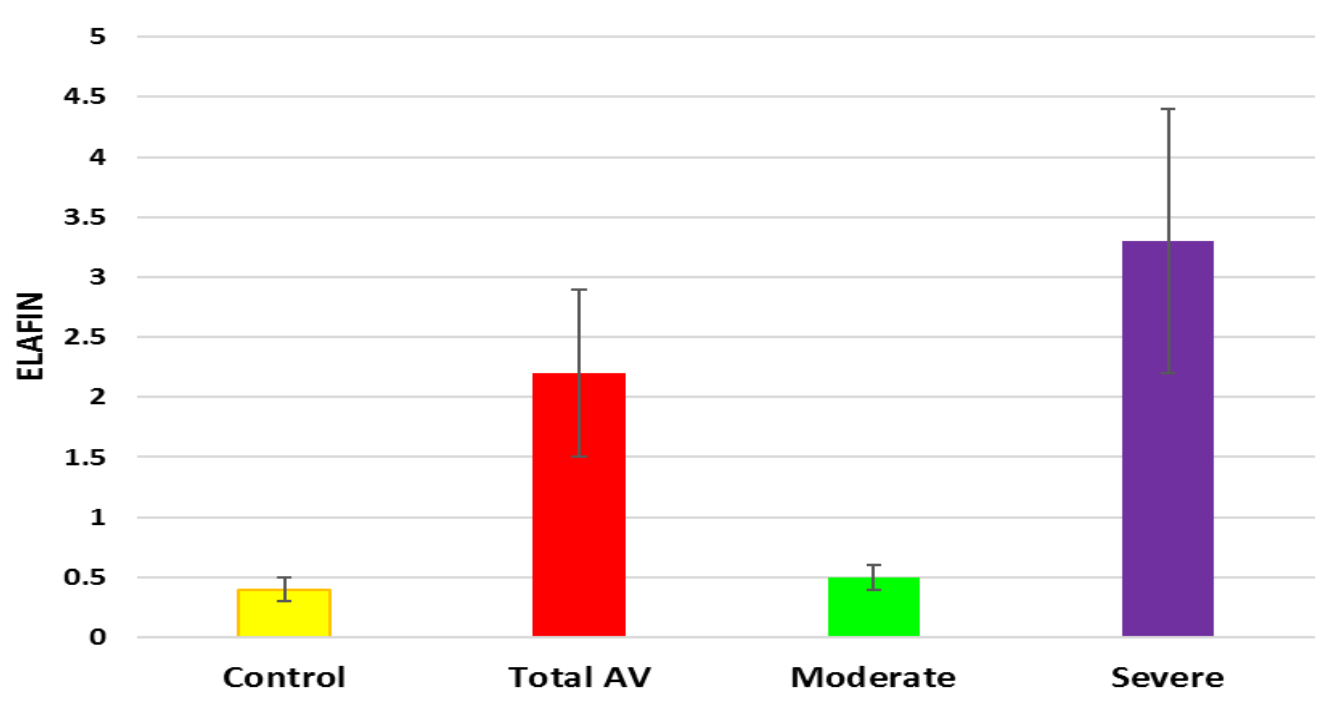

Fig (2) Elafin concentration in all studied groups.

Receiver operating characteristic (ROC) curve of Elafin was conducted for discrimination between acne vulgaris cases and control groups. Elafin showed excellent AUC (AUC=0.928, p<0.001). At cut off value of 0.43 , sensitivity was $92.5 \%$, specificity was $82.5 \%$, PPV was $84.1 \%$, NPV was $91.7 \%$, accuracy was $87.5 \%$. CRP showed good AUC (AUC $=0.918$, $\mathrm{p}<0.001$ ). At cut off value of 6 , sensitivity was $82.5 \%$, specificity was 95\%, PPV was 94.3\%, NPV was $84.4 \%$, accuracy was $88.8 \%$. Fig (4).

\section{Discussion}

One interesting finding from our analysis of serum elafin is that we detected significant higher serum levels of Elafin compared to control group, and correlated with the severity of the acne , and it wasn't 
measured before.

Also it was interesting to found that Elafin concentration increased significantly $(p>0.001)$ with scar formation.

This finding is in line with another dermatological disease in [8], who mentioned that elafin levels correlated well with the clinical state of psoriasis and its severity.

In liu et al study [9], indicated that (77.3\%) an increase expression of Elafin compared with $59.1 \%$ matched adjacent noncancerous tissues.

This positive correlation between elafin and the severity of the disease may be attributed to the worsening of acne linked to an imbalance between neutrophil activation products "as elastase and protease" and their inhibitors "as elafin",

In wang et al study [10] showed high serum elafin levels were associated with a significantly elevated risk of intestinal stricture. Also ulcerative colitis (UC) patients had high serum elafin levels in Crohn's disease (CD) patients.

In wang et al sttudy [10] Circulating elafin has moderate sensitivity and specificity in indicating clinical disease activity in Crohn's disease and ulcerative colitis patients. The accuracies of elafin in indicating clinical disease activities $(\mathrm{AUC}=0.716$ in Crohn's disease and AUC $=0.723$ in ulcerative colitis.

The excellent performance of the measured markers in our patients together with the absence of a significant difference in the diagnostic ability of them suggests that both markers had excellent diagnostic performance in evaluating acne vulgaris but have the same discriminator power between acne and healthy control subjects.

\section{Conclusion}

Elafin acts as an alarm anti protease; the measurement of its levels in serum of patients with acne vulgaris provides a tool for monitoring disease activity and could be used as an indicator of the disease severity and inflammatory state.

\section{References}

[1] K. Bhate and H. C. Williams, "Epidemiology of acne vulgaris," Br. J. Dermatol, Vol.168, PP. 474-485, 2013.

[2] H. Gollnick et al., "Management of acne: a report from a Global Alliance to Improve Outcomes in Acne," J. Am. Acad. Dermatol, Vol.49, PP. S1-S37, 2003.

[3] M.-C. Tsai, W. Chen, Y.-W. Cheng, C.-Y. Wang, G.-Y. Chen, and T.-J. Hsu, "Higher body mass index is a significant risk factor for acne formation in schoolchildren," Eur. J. Dermatology, Vol. 16, PP. 251-253, 2006.

[4] L. A. Zaleski-Larsen, S. G. Fabi, T. McGraw, and M. Taylor, "Acne scar treatment: a multimodality approach tailored to scar type," Dermatologic Surg, Vol.42, PP. S139-S149, 2016.

[5] L. H. Christensen, "Host tissue interaction, fate, and risks of degradable and nondegradable gel fillers," Dermatologic Surg, Vol.35, PP. 16121619, 2009.

[6] D. M. Small et al., "A functional variant of elafin with improved anti-inflammatory activity for pulmonary inflammation," Mol. Ther, Vol.23, PP. 24-31, 2015.

[7] L. Shaw and O. Wiedow, "Therapeutic potential of human elafin.” Portland Press Ltd., vol.1, PP. 65-87, 2011.

[8] I. Elgharib, S. A. Khashaba, H. H. Elsaid, and M. M. Sharaf, "Serum elafin as a potential inflammatory marker in psoriasis," Int. J. Dermatol, Vol.58, PP. 205-209, 2019.

[9] Y. Liu, Y. Tian, T. Wu, Y. Dai, W. Wang, and G. Teng, "High Expression and Clinical Significance of Elafin in Colorectal Cancer," Gastroenterol. Res. Pract, Vol.2019, PP. 23-54, 2019.

[10] J. Wang et al., "High circulating elafin levels are associated with Crohn's disease-associated intestinal strictures," PLoS One, Vol. 15, P. e0231796, 2020. 